Sharif University of Technology
Scientia Iranica
SCIENTIA
IRAN I CA

\title{
Determination of ship maneuvering hydrodynamic coefficients using system identification technique based on free-running model test
}

\author{
S. Hajizadeh, M.S. Seif* and H. Mehdigholi \\ Department of Mechanical Engineering, Sharif University of Technology, Center of Excellence in Hydrodynamics and Dynamics of \\ Marine Vehicles, Tehran, Iran.
}

Received 12 April 2015; received in revised form 18 August 2015; accepted 26 October 2015

\author{
KEYWORDS \\ Maneuvering; \\ Modeling; \\ Displacement hull; \\ Hydrodynamic \\ coefficient; \\ System identification \\ technique.
}

\begin{abstract}
In the recent years, different mathematical models have been suggested for maneuvering of displacement vessels that are capable of estimation of vessel maneuvers with acceptable precision. These mathematical models are based on determined hydrodynamic coefficients and their accuracy depends on the known coefficients used to solve the mathematical model. System identification methods are developed to calculate these coefficients utilizing input and output data obtained from different sources. In this research, a 4.36-m model of KRISO Container Ship (KCS) displacement vessel has been manufactured by fiberglass, and the maneuver turning tests have been carried by selfpropulsion method. A 3DM-GX1 sensor, together with a protractor and Global Positioning System (GPS), has been used to measure the yaw and rudder angle, position, linear accelerations, and angular velocities of the vessel in different times. The hydrodynamic coefficients in the mathematical model are determined by the Extended Kalman filter method. Then, the mathematical model is solved and different maneuvers are simulated by coefficients calculated from the experiments. Simulations are validated by model tests. The mathematical model and hydrodynamic coefficients presented in this paper can be applied for optimization of ship maneuvering performance and course control purposes.

(C) 2016 Sharif University of Technology. All rights reserved.
\end{abstract}

\section{Introduction}

Predicting maneuvering performance of the ship has been a major subject of study in hydrodynamics of ships. Ship maneuvers are extremely nonlinear and unsteady phenomena due to dynamic coupling and hydrodynamic viscous effects. Due to the limitations of the analytical methods in predicting maneuver of the ship, experimental formulae based on the model tests have been the main method in use. The men-

\footnotetext{
*. Corresponding author. Tel.: +98 2166165549 ;

Fax: +982166165563

E-mail address: seif@sharif.edu (M.S. Seif)
}

tioned method is based on mathematical models and maneuvering coefficients, which are determined by experiments or by analytical methods.

Predicting ship maneuvering in calm water is an important topic; however, it is very expensive to run numerous maneuvering simulations using Computational Fluid Dynamics (CFD). Despite their short computation time, system-based simulations need many captive model tests to estimate the hull and rudder maneuvering coefficients used in the system-based mathematical model.

System-Based (SB) and CFD methods are major simulation methods to predict ship maneuverability. Computation time of the SB simulation is much lower 
than that of CFD, since such methods need only to solve the equations of motion using a prescribed mathematical model and maneuvering coefficients. SB simulation requires approximately few minutes for one free-running trial, while CFD needs a few weeks or a month depending on the turbulence and propulsion modeling and size of grids. However, there are many issues in derivation of most accurate mathematical models and maneuvering coefficients. In most cases, captive model planar motion mechanism or rotating arm/circular motion tests are used, which require many static and dynamic conditions.

Ship motion at sea is a complex system with characteristics of highly nonlinear nature, long time delay, and time-varying dynamics. There are three kinds of mathematical models of ship maneuvering motion, i.e. Abkowitz model, Maneuvering Mathematical Modeling Group (MMG) model, and response model [1].

Recently, a new maneuvering model was developed from first principles of low aspect-ratio aerodynamic theory and Lagrangian mechanics [2]. Meanwhile, the MMG expanded a mathematical model which explicitly included the individual open water characteristics of the hull/propeller/rudder and their interactions [3]. The MMG model has shown good results for maneuvering in calm water and qualitative results for extensions of wave conditions [4].

Ship maneuvering models are the keys to the research on ship maneuverability, design of ship motion control system, and development of ship handling simulators [5]. For various frames of ship maneuvering models, determining the parameters of the models is always a tedious task. The usual approach to determine the ship model parameters is the ship model test. Ship model test is a reliable and accurate method for this purpose. The test is, however, expensive and time-consuming, and usually dependent on some specific model frame, which limits the application of the valuable data [6].

System identification techniques are developed in control engineering to build mathematical models for dynamical systems often using maneuvering coefficients based on experimental data. The Least Square (LS) is one of the simplest, but the Extended Kalman Filtering (EKF) is one of the most widely used methods in engineering [7]. For ship hydrodynamics applications, the advantage of system identification is that all the maneuvering coefficients can be estimated by one or a few free-running trials as opposed to numerous captive model tests.

The Kalman filter is an efficient recursive filter that estimates the state of a dynamic system from a series of noisy measurements. It is able to provide solutions to what probably are the most fundamental problems in control theory [8]. EKF is the nonlinear version of the Kalman filter and is often considered the de facto standard in the theory of nonlinear state estimation. EKF is widely used in areas of state estimation, object tracking, and navigation [9].

As the system identification method is an approach to find a model of unknown system, estimation of maneuvering coefficients can be said as an application of system identification. Several schemes were suggested for the estimation of maneuvering coefficients [10]. Abkowitz employed EKF using full-scale trial data and the Abkowitz mathematical model [11]. Rhee and Kim employed EKF for SB free-running trial data and the MMG mathematical model to find the best trial type for system identification. The maneuvering coefficients reconstructed from the largeangle zigzag test showed the smallest error with the original coefficients [12]. Sung et al. presented simulations with an Abkowitz model and an MMG model using a system identification technique for identifying the derivatives [13]. They concluded that reasonable results were obtained from a considerably reduced number of dynamic test runs compared with a traditional approach.

Zhang and Zou employed support vector machine, one of the artificial intelligence methods, for SB freerunning trial data (zigzag test) using the Abkowitz mathematical model [14]. This research showed that coefficients had close agreement with the original maneuvering coefficients.

In the present paper, a model of KCS was manufactured in the Marine Engineering Laboratory at Sharif University of Technology and the free-running tests were performed on it in an open calm lake. Turning test was performed in free-running mode and meanwhile, the model accelerations were measured for the surge, sway, and yaw motions. Then the maneuvering of the ship was simulated by using the mathematical equations of 3 degrees of freedom of Abkowitz and the obtained results of the selfpropulsion tests. The advanced Kalman filter method was used to estimate the hydrodynamic coefficients of this ship and all of the maneuvering coefficients of the ship were derived. Having derived the hydrodynamic coefficients, the turning maneuver was simulated and compared with the experimental results. This paper shows that the maneuvering coefficients obtained with free-running trial data have high accuracy. They can be used in the mathematical model to simulate the ship maneuvering that has close agreement with the experimental result.

\section{Displacement hull maneuvering equations}

It is convenient to assume a constant wetted surface in analysis of seakeeping and maneuvering for displacement hulls.

In Figure 1, $u$ is the surge velocity, $v$ is sway 


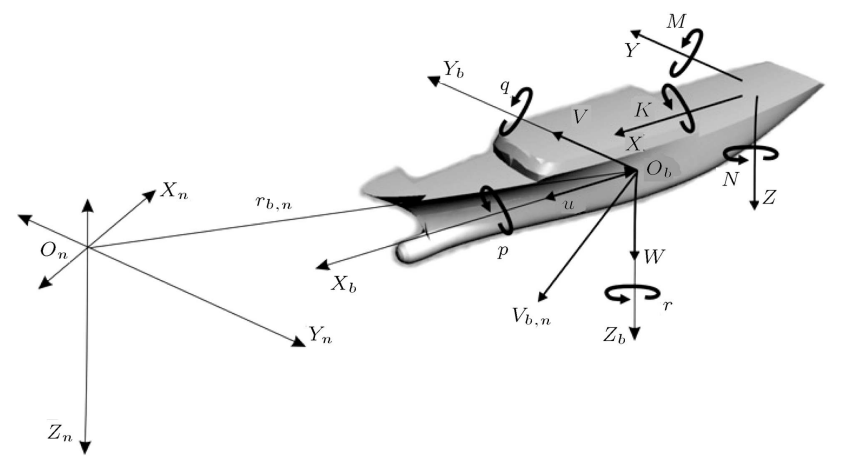

Figure 1. Fixed and moving reference frames of ship motion [15].

velocity, $w$ is heave velocity, $p$ is roll angular velocity, $q$ is pitch angular velocity, $r$ is yaw angular velocity, $X$ is the force exerted on the hull in the surge direction, $Y$ is exerted force in sway direction, $Z$ is exerted force in heave direction, $K$ is the roll moment exerted on the hull, $M$ is the pitch moment, $N$ is yawing moment, $I$ is mass moment of inertia, and $\omega$ is vessel angular velocity vector [16].

$$
\begin{aligned}
& X=m\left(\dot{u}+\omega q_{b}-v r_{b}\right), \\
& Y=m\left(\dot{v}+u r_{b}-w p_{b}\right), \\
& Z=m\left(\dot{w}+v p_{b}-u q_{b}\right), \\
& K=\frac{d}{d t}\left(I_{x x} \omega_{x}-I_{x y} \omega_{y}-I_{x z} \omega_{z}\right) \\
& -r_{b}\left(I_{y y} \omega_{y}-I_{y z} \omega_{z}-I_{x y} \omega_{x}\right) \\
& +q_{b}\left(I_{z z} \omega_{z}-I_{x z} \omega_{x}-I_{y z} \omega_{y}\right), \\
& M=\frac{d}{d t}\left(I_{y y} \omega_{y}-I_{y z} \omega_{z}-I_{x y}-\omega_{x}\right) \\
& -p_{b}\left(I_{z z} \omega_{z}-I_{x z} \omega_{x}-I_{y z} \omega_{y}\right) \\
& +r_{b}\left(I_{x x} \omega_{x}-I_{x y} \omega_{y}-I_{x z} \omega_{z}\right), \\
& N=\frac{d}{d t}\left(I_{z z} \omega_{z}-I_{x z} \omega_{x}-I_{y z}-\omega_{y}\right) \\
& -q_{b}\left(I_{x x} \omega_{x}-I_{x y} \omega_{y}-I_{x z} \omega_{z}\right) \\
& +p_{b}\left(I_{y y} \omega_{y}-I_{y z} \omega_{z}-I_{x y} \omega_{x}\right) .
\end{aligned}
$$

In calm water, if surge motion is considered of first order, sway and yaw motions would be of the second order, because the forward speed is so larger than yaw and sway motion velocities. Moreover, the heave, roll, and pitch motions are of the third order. Thus, the vessel motions in calm water can be reduced to three degrees of freedom of surge, sway, and yaw:

$$
\left\{\begin{array}{l}
m\left(\dot{u}-v r-x_{G} r^{2}\right)=X_{G} \\
m\left(\dot{v}_{G}+x_{G} \dot{r}+u r\right)=Y_{G} \\
I_{z} \dot{r}+m x_{G}(\dot{v}+u r)=N_{G}
\end{array}\right.
$$

It can be assumed that the center of coordinates is considered on vessel center of gravity. Thus, the equations of motion are reduced to Eq. (3):

$$
\left\{\begin{array}{l}
m\left(\dot{u}_{G}-v_{G} r\right)=X_{G} \\
m\left(\dot{v}_{G}+u_{G} r\right)=Y_{G} \\
I_{z} \dot{r}=N_{G}
\end{array}\right.
$$

Eq. (2) is the equation of motion with the center of gravity of ship; $G$ and the notations of $u_{G}, v_{G}$, and $r$ are velocity components at the center of gravity of ship; $X_{G}, Y_{G}$, and $N_{G}$ represent the hydrodynamic forces and moment acting on $G$.

Hydrodynamic forces of surge, sway, and moment of yaw are considered as:

$$
\left\{\begin{aligned}
X_{G}= & X_{u} u+X_{v v} v^{2}+X_{r r} r^{2}+X_{\delta \delta} \delta^{2} \\
& +X_{v \delta} v \delta+X_{r \delta} r \delta+X_{u n} u n+X_{n n} n^{2} \\
Y_{G}= & Y_{v} v+Y_{r} r+Y_{\delta} \delta \\
N_{G}= & N_{v} v+N_{r} r+N_{\delta} \delta
\end{aligned}\right.
$$

By substituting the above equations in Eq. (3), we have:

$$
\left\{\begin{array}{l}
m(\dot{u}-v r)=X_{u} u+X_{v v} v^{2}+X_{r r} r^{2}+X_{\delta \delta} \delta^{2} \\
\quad+X_{v \delta} v \delta+X_{r \delta} r \delta+X_{u n} u n+X_{n n} n^{2} \\
m(\dot{v}+u r)=Y_{v} v+Y_{r} r+Y_{\delta} \delta \\
I_{z} \dot{r}=N_{v} v+N_{r} r+N_{\delta} \delta
\end{array}\right.
$$

$\delta$ is the rudder angle and $n$ is the propeller RPM of the model.

By rewriting the above equation, we have:

$$
\left\{\begin{aligned}
\dot{u}= & \frac{X_{u}}{m} u+\frac{X_{v v}}{m} v^{2}+\frac{X_{r v}}{m} r^{2}+\frac{X_{\delta \delta}}{m} \delta^{2}+m v r \\
& +\frac{X_{v \delta}}{m} v \delta+\frac{X_{r \delta}}{m} r \delta+\frac{X_{u n}}{m} u n+\frac{X_{n n}}{m} n^{2} \\
\dot{v}= & \frac{Y_{v}}{m} v+\frac{Y_{r}}{m} r+\frac{Y_{\delta}}{m} \delta-m u r \\
\dot{r}= & \frac{N_{v}}{I_{z}} v+\frac{N_{r}}{I_{z}} r+\frac{N_{\delta}}{I_{z}} \delta
\end{aligned}\right.
$$

in which keeping $\frac{X_{u}}{m}=a_{1}, \frac{X_{v v}}{m}=a_{2}, \ldots, \frac{Y_{v}}{m}=b_{1}, \ldots$, 
$\frac{N_{v}}{I_{z}}=c_{1}, \ldots$ and rewriting the remaining part give:

$$
\begin{aligned}
\dot{u}= & a_{1} u(t)+a_{2} v(t)^{2}+a_{3} r(t)^{2}+a_{4} \delta(t)^{2} \\
& +a_{5} v(t) r(t)+a_{6} v(t) \delta(t)+a_{7} r(t) \delta(t) \\
& +a_{8} u(t) n(t)+a_{9} n(t)^{2}, \\
\dot{v}= & b_{1} v(t)+b_{2} r(t)+b_{3} \delta(t)+b_{4} u(t) r(t), \\
\dot{r}= & c_{1} v(t)+c_{2} r(t)+c_{3} \delta(t),
\end{aligned}
$$

where $a_{i}(i=1,2, \ldots, 9), b_{i}(i=1,2,3,4)$, and $c_{i}(i=$ $1,2,3)$ are model parameters that should be determined through system identification.

\section{Free-running maneuvering model test of displacement hull}

A 4.36-m model of KCS is manufactured and equipped with a rudder and propeller and the meticulous measurement devices are installed on it. Then, the various standard maneuvering tests are performed. The main dimensions of full-scale KCS are shown in Table 1. Table 2 represents the corresponding model parameters.

The propeller RPM of model is not measured during the test. RPM of the propeller is changed until the speed of the model reaches the service speed; also, rudder angle of model is 35 degrees in this test.

The experimental site is Azadi Lake and the environmental information in average at that time is: temperature $=23^{\circ} \mathrm{C}$, wind speed $=1.21 \mathrm{~m} / \mathrm{s}$ and current speed $=0.22 \mathrm{~m} / \mathrm{s}$.

The KCS hull form is presented in Figure 2 and the manufactured model together with its rudder and propeller is shown in Figure 3. Figure 4 and Figure 5 show the maneuvering model test in calm water of open lake.

\section{Measurement equipment}

\subsection{DM-GX1 sensor}

3DM-GX1 sensor rotates in 360 degrees and gives

\begin{tabular}{|c|c|}
\hline Scale & 52.667 \\
\hline \multicolumn{2}{|c|}{ Main particulars } \\
\hline $\operatorname{Lpp}(\mathrm{m})$ & 4.3671 \\
\hline Lwl (m) & 4.4141 \\
\hline $\mathrm{Bwl}(\mathrm{m})$ & 0.6114 \\
\hline $\mathrm{D}(\mathrm{m})$ & 0.3608 \\
\hline $\mathrm{T}(\mathrm{m})$ & 0.2051 \\
\hline Displacement $\left(\mathrm{m}^{3}\right)$ & 0.3562 \\
\hline $\mathrm{S} \mathrm{w} / \mathrm{o}$ rudder $\left(\mathrm{m}^{2}\right)$ & 3.4357 \\
\hline $\mathrm{CB}$ & 0.651 \\
\hline $\mathrm{CM}$ & 0.984 \\
\hline $\operatorname{LCB}(\%)$, fwd + & -1.48 \\
\hline \multicolumn{2}{|c|}{ Rudder } \\
\hline Type & semi-balanced horn rudder \\
\hline $\mathrm{S}$ of rudder $\left(\mathrm{m}^{2}\right)$ & 0.0415 \\
\hline Lat. area $\left(\mathrm{m}^{2}\right)$ & 0.0196 \\
\hline Turn rate $(\mathrm{deg} / \mathrm{s})$ & 16.8 \\
\hline \multicolumn{2}{|c|}{ Propeller } \\
\hline Type & $\mathrm{CP}$ \\
\hline No. of blades & 5 \\
\hline $\mathrm{D}(\mathrm{m})$ & 0.150 \\
\hline $\mathrm{P} / \mathrm{D}(0.7 \mathrm{R})$ & 1.300 \\
\hline $\mathrm{Ae} / \mathrm{A} 0$ & 0.700 \\
\hline Rotation & Right hand \\
\hline Hub ratio & 0.227 \\
\hline \multicolumn{2}{|c|}{ Test condition } \\
\hline $\mathrm{T}(\mathrm{m})$ & 0.205 \\
\hline Disp. $\left(\mathrm{m}^{3}\right)$ & 0.356 \\
\hline $\mathrm{S}\left(\mathrm{m}^{2}\right)$ incl. rudder & 3.477 \\
\hline LCG (m) & 2.119 \\
\hline $\mathrm{GM}(\mathrm{m})$ & 0.011 \\
\hline $\mathrm{ixx} / \mathrm{B}$ & 0.40 \\
\hline $\mathrm{izz} / \mathrm{Lpp}$ & 0.25 \\
\hline \multicolumn{2}{|c|}{ Service speed } \\
\hline $\mathrm{U}(\mathrm{m} / \mathrm{s})$ & 1.701 \\
\hline Fn (based on Lpp) & 0.26 \\
\hline
\end{tabular}
the direction in matrix, quaternion, and Eulerian

Table 1. Main dimensions of KCS (full scale).

\begin{tabular}{cc}
\hline LBP $(\mathrm{m})$ & 230.0 \\
LWL $(\mathrm{m})$ & 232.5 \\
$\mathrm{~B}(\mathrm{~m})$ & 32.2 \\
$\mathrm{D}(\mathrm{m})$ & 19.0 \\
$\mathrm{~T}(\mathrm{~m})$ & 10.8 \\
Displacement & 52030 \\
CB & 0.651 \\
$\mathrm{CM}$ & 0.985 \\
LCB \% fwd. & -1.48 \\
\hline
\end{tabular}

Table 2. Main dimensions of KCS (model scale).

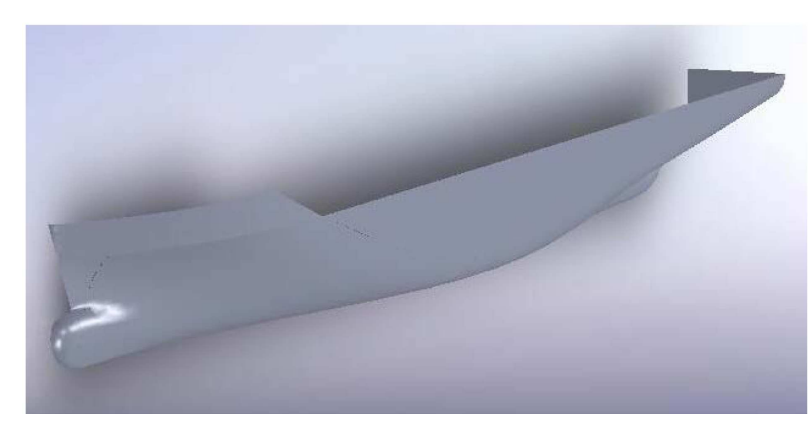

Figure 2. KCS hull form solidwork simulation. 


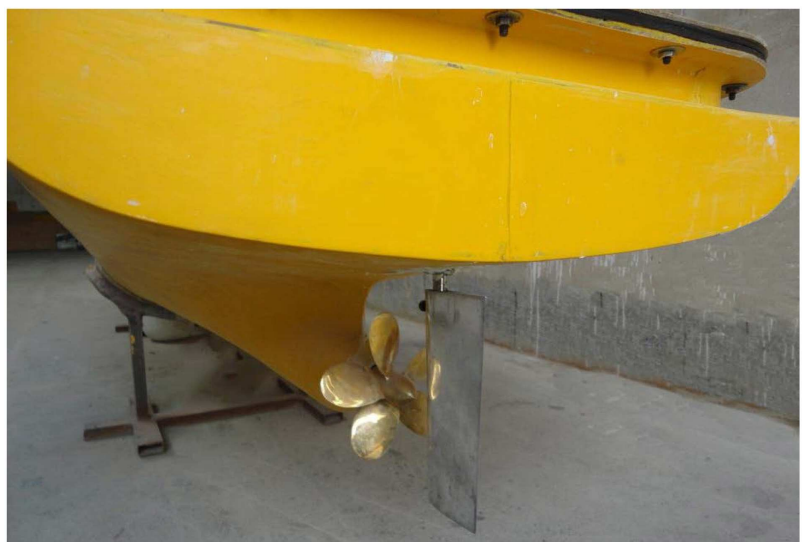

Figure 3. The manufactured model together with its rudder and propeller.

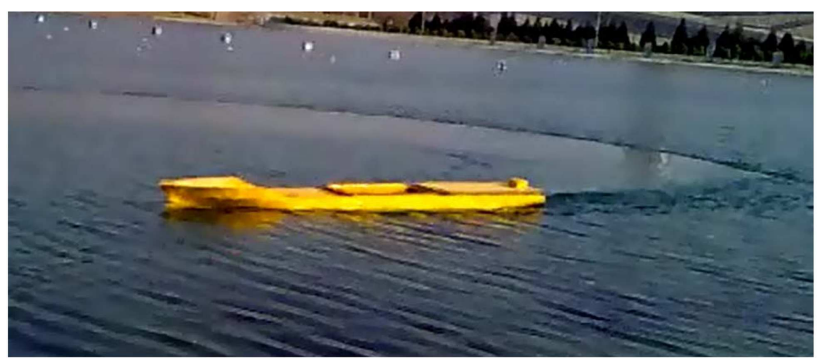

Figure 4. Turning maneuvering model test of KCS hull.

forms. The digital output shows the temperature and perpendicular accelerations with frequency of $350 \mathrm{~Hz}$. The output filter parameters and its conditions can be changed by user.

One of the advantages of the used sensor is the processing and filtering of the data by itself. Also, it can be calibrated in constant magnetic fields.

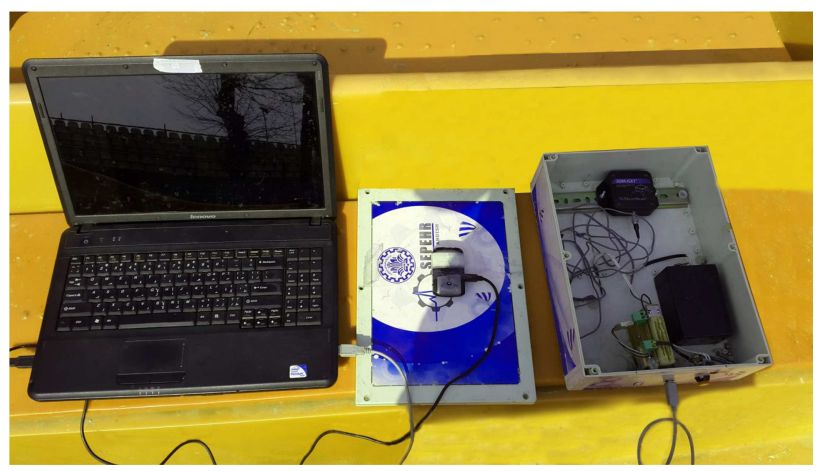

Figure 5. A photo of the data recording model.

Furthermore, this sensor is small, light, and lowconsumption. It can be utilized for underwater and unmanned vehicles, robotics, moving cameras, moving radio antennas, hydraulic lift systems, etc. The specifications of this sensor are shown in Table 3. A photo of the data recording Module (consisting of 3DM-GX1 sensor, GPS, and Laptop) is shown in Figure 5.

This sensor utilizes the triaxial gyros to track dynamic orientation and the triaxial DC accelerometers along with the triaxial magnetometers to track static orientation. The embedded microprocessor contains a unique programmable filter algorithm, which blends these static and dynamic responses in real time. This kind of data processing leads to a reference response with omitted deviations due to vibrations and fast motions.

\subsection{Global positioning system}

The GPS is supported by 24 satellites on Earth's orbit. The GPS satellites orbit the Earth twice every day and send signals containing information.

Table 3. Specifications of 3DM-GX1 sensor.

\begin{tabular}{ll}
\hline Orientation range (pitch, roll, yaw) & $360^{\circ}$ all axes (orientation matrix, quaternion) \\
& $\pm 90^{\circ}, \pm 180^{\circ}, \pm 180^{\circ}$ (Euler angles) \\
& Gyros: $\pm 300^{\circ} / \mathrm{sec} \mathrm{FS}$ \\
& Accelerometers: $\pm 5 \mathrm{~g} \mathrm{FS}$ \\
& Magnetometers: \pm 1.2 Gauss FS \\
Sensor range & 16 bits \\
& $0.2 \%$ \\
A/D resolution of 16 bits & $0.010 \mathrm{~g}$ \\
Accelerometer nonlinearity & $0.2 \%$ \\
Accelerometer bias stability & $0.7^{\circ} / \mathrm{sec}$ \\
Gyro nonlinearity & $<0.1^{\circ}$ minimum \\
Gyro bias stability & $0.20^{\circ}$ \\
Orientation resolution & $\pm 0.5^{\circ}$ typical for static test conditions \\
Repeatability & $\pm 2.0^{\circ}$ typical for dynamic (cyclic) test conditions \\
& and for arbitrary orientation angles \\
\hline
\end{tabular}


Table 4. Main specifications of the used GPS.

\begin{tabular}{cc}
\hline Specifications & Description \\
\hline Accuracy & Position $5 \mathrm{~m} \mathrm{CEP}$ \\
& Velocity $0.1 \mathrm{~m} / \mathrm{sec}$ \\
Startup & $<1 \mathrm{sec}$ hot start \\
Time (average) & $<30 \mathrm{sec}$ cold start \\
Sensitivity & $-155 \mathrm{dBm}$ acquisition \\
& $-160 \mathrm{dBm}$ tracking \\
Protocol & NMEA-0183 V3.01 \\
& $4800 / 9600 / 19200 / 38400$ baud, 8, N, 1 \\
Input voltage & $3.8 \mathrm{~V} \sim 8.0 \mathrm{~V}$ DC input \\
\hline
\end{tabular}

A GPS receiver should receive signals of 3 satellites in order to determine the two (latitude and longitude) positions of an object. The signal of four or more satellites can determine three positions (latitude, longitude, and height). Also, GPA can be used for speed measurement, orientation, searching, etc. The main specifications of the used module are mentioned in Table 4.

\section{System identification method}

Hydrodynamic derivatives are usually obtained from the measured hydrodynamic forces. In the system identification method, they can be obtained from the measured ship motion and applied rudder angle. Since the ship motion is identified accurately by this method, it is well used for the design of control and navigation systems. System identification method can be defined as a systematic approach to find a model of unknown system from the given input-output data. For the successful system identification, three items should be properly selected or designed: mathematical model of a system, input-output data, and parameter estimation scheme.

Modeling and simulation is the key approach of analysis in all branches of science and technology. Modeling is the quantification of a relation between input and output of the system through a mathematical description. Simulation is the real-time representation of the mathematical model through digital computations to simulate the reality. Thus, modeling is the derivation of a mathematical model such that when an input signal is applied to it, the outputs would be the same as the real system. System identification is a method of derivation of mathematical model based on experiment [17]. The block diagram of the system identification method is shown in Figure 6. System identification method is executed through five major

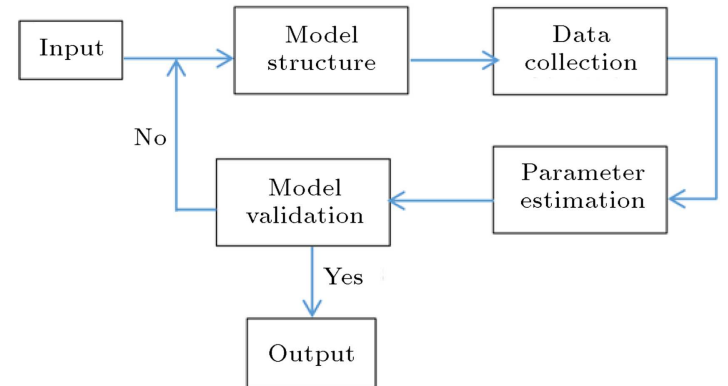

Figure 6. Block diagram of the system identification method.

steps:

1. Definition of system, inputs, and resultant outputs;

2. Definition of structure (model) for the system;

3. Experimentation and gathering of input and output data;

4. Estimation of parameters needed;

5. Evaluation of the model.

\subsection{Advanced Kalman filter}

Usually, noise sample is observed in the process of data sampling. Thus, the most appropriate procedures are based on probabilistic and statistical data and Kalman filter is one of the recognized methods used. The extended Kalman filter method optimizes the objective parameters and state variables statically. Therefore, the extended Kalman filter method does not need input acceleration data that is usually associated with noise in experimental data; this would be a great advantage in application. Here, the extended Kalman filter method is used to obtain ship maneuvering hydrodynamic coefficients based on the results obtained from free-running model tests and analytical Eq. (3). The details of extended Kalman filter are provided in Appendix A. 


\section{Calculation of hydrodynamic coefficients}

The values of surge, sway, and yaw velocities measured from free-running maneuvering model tests are applied to extended Kalman filter as input and this method calculates the hydrodynamic coefficients in such a way that minimum error is obtained when compared to input velocity values. The values of velocities calculated by extended Kalman filter are shown in Figure 7.

Figures 8 and 9 show some of the hydrodynamic coefficients used in Eq. (3) that are calculated by extended Kalman filter method and the values of all calculated hydrodynamic coefficients are presented in Table 5. The comparison between experimental $U$ of the experiments and the Kalman filter is shown in Figure 10.
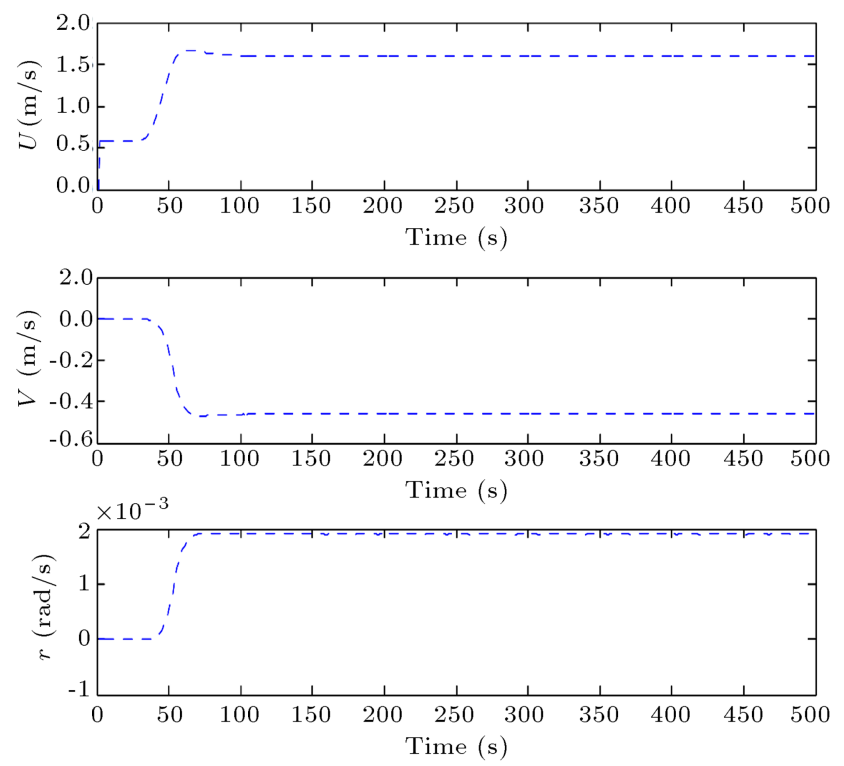

Figure 7. Simulation of ship linear and angular velocities. Rudder angle of model was $35^{\circ}$.
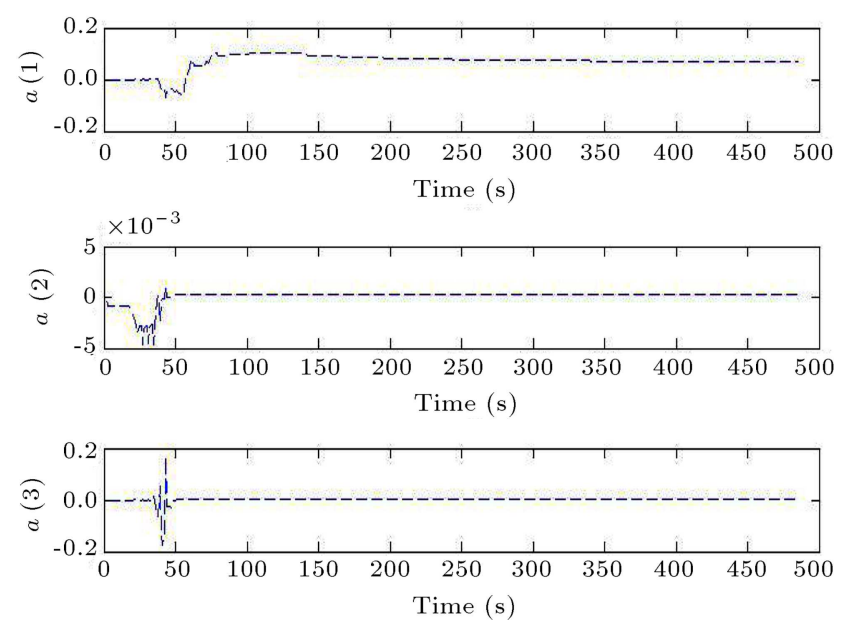

Figure 8. $a_{1}, a_{2}$ and $a_{3}$ coefficients calculated by system identification method.

\section{Simulation of turning circle}

The ships should have enough turning ability in order to avoid collision and should show enough stability; also, turning circle diameter should not exceed certain values. Using software simulation in maneuver analysis of vessels is an appropriate method to evaluate their maneuverability in the lowest possible time with applicable results. The maneuverability of a vessel can be investigated via numerical simulations if
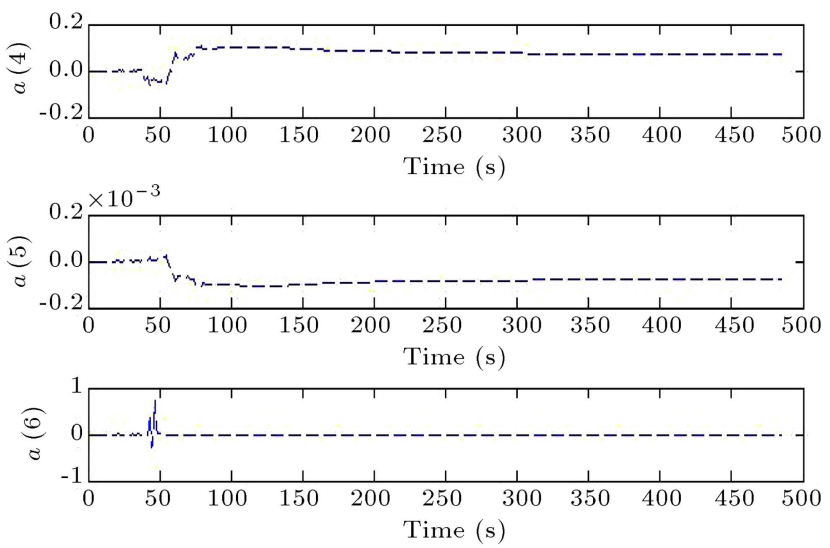

Figure 9. $a_{4}, a_{5}$, and $a_{6}$ coefficients calculated by system identification method.

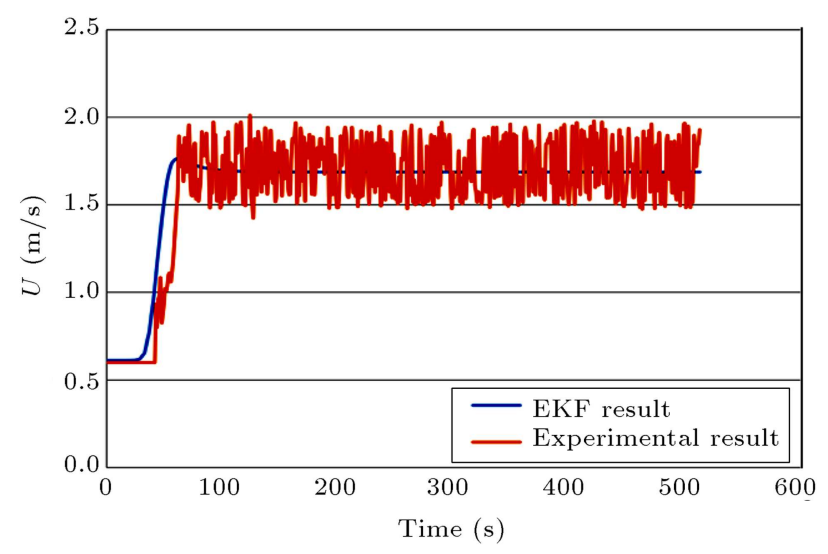

Figure 10. Comparison between speed of the experiments and EKF.

Table 5. Hydrodynamic coefficients calculated by system identification method.

\begin{tabular}{cccc}
\hline Parameter & Value & Parameter & Value \\
\hline$a_{2}$ & $2.85 \times 10^{-4}$ & $a_{1}$ & $6.75 \times 10^{-2}$ \\
$a_{4}$ & $6.76 \times 10^{-2}$ & $a_{3}$ & $1.2 \times 10^{-3}$ \\
$a_{6}$ & $-2.94 \times 10^{-4}$ & $a_{5}$ & $-7.605 \times 10^{-4}$ \\
$a_{8}$ & $-2.865 \times 10^{-2}$ & $a_{7}$ & $8.984 \times 10^{-3}$ \\
$b_{1}$ & $-3.67 \times 10^{-3}$ & $a_{9}$ & $4.791 \times 10^{-3}$ \\
$b_{3}$ & $4.616 \times 10^{-3}$ & $b_{2}$ & 7.2717 \\
$c_{1}$ & $-4.5 \times 10^{-4}$ & $b_{4}$ & -2.38834 \\
$c_{3}$ & $-2.8 \times 10^{-4}$ & $c_{2}$ & $-9.987 \times 10^{-2}$ \\
\hline
\end{tabular}




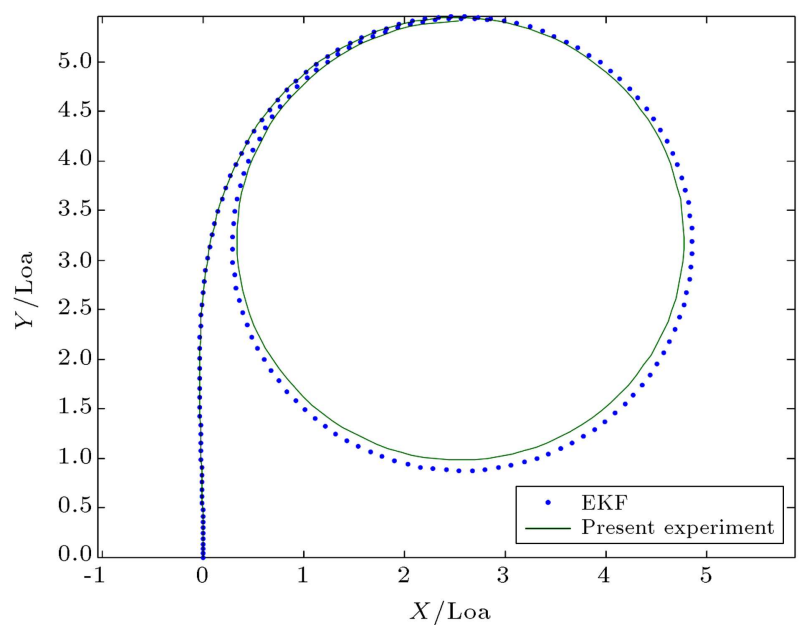

Figure 11. Comparison of the turning circle measured in free-running model test and simulation by system identification method.

some experimental data such as hydrodynamic coefficients, propulsion system, and rudder characteristics are available. In this section, the turning maneuver of the KCS is simulated by solving the maneuvering equations numerically. The test is repeated for both rudder to port and rudder to starboard directions.

Considering that the essential hydrodynamic coefficients for Eq. (3) were determined by self-propulsion test together with system identification method and noting that the data for propeller hydrodynamics of the vessel is available, this equation is solved numerically by substituting the mentioned parameters in the equation. By solving this equation, the velocity component is determined. Also, the positions of the vessel and yawing angle are determined by integrating the velocity in time. The comparison of turning circles measured in free-running model test and simulation by system identification method is shown in Figure 11.

As the hydrodynamic coefficients are determined by the results obtained from model experiments through system identification method, these coefficients can be applied to complete the mathematical model and simulate the various ship maneuvers. The turning circle is simulated in the following by a mathematical model developed from free-running model test results. It is essential to solve the equations of motion for rudder deflected to port and starboard side.

In the starboard turning case, the rudder angle was set on $+35^{\circ}$. Also, the equations were solved. The results of this simulation are shown in Figure 12. As it can be seen, there is a good agreement between numerical and experimental results. However, it should be checked if the drift angle of the ship during turning simulation is in agreement with turning trajectory. The accurate position of the model is determined using yawing angles and rotated by rotating matrix. The

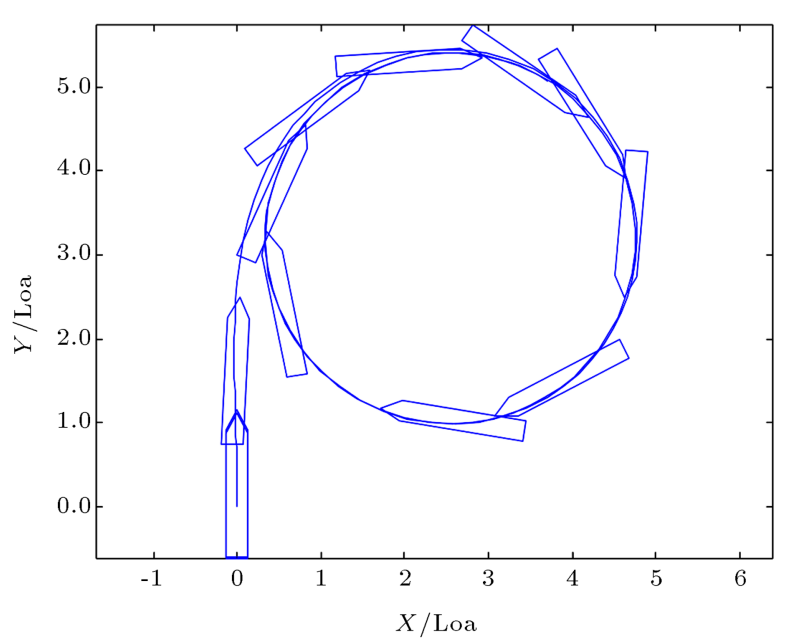

Figure 12. Simulation of starboard turning circle with rudder angle set on $+35^{\circ}$.

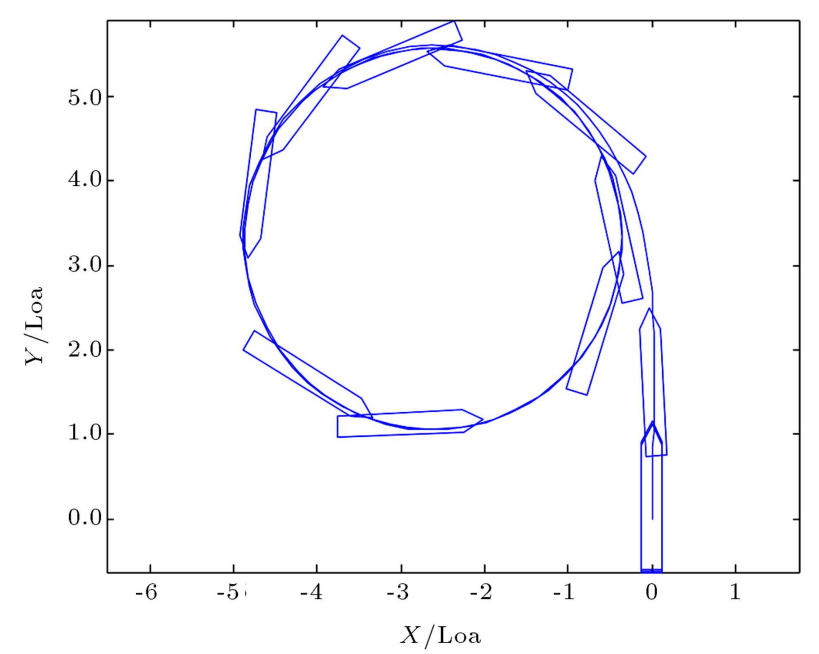

Figure 13. Simulation of port turning circle with rudder angle set on $-35^{\circ}$.

position coincides with turning circle in different times. The heading change of the vessel is shown in Figure 12, too, and it can be seen that it matches with the turning trajectory quite well. Therefore, the maneuverability of a vessel can be assessed using this approach with a great accuracy.

The developed mathematical model is symmetric for turning to port or starboard side. Thus, it should be checked if the mathematical model leads to the same behavior for turning to port side. All the maintained procedure is carried out for the case of port turning maneuver with $35^{\circ}$ rudder angle (see Figure 13 ).

The ship model in this study has been considered under calm and deep water conditions. In the actual navigational situation, there are many kinds of environmental forces such as wind, wave, and current. Important sources of error in this study are simplification in the employed mathematical model, such as eliminating the higher orders of coefficients, and ignoring forces due 
to the environmental condition. Uncertainties are due to environmental condition in the experiments, scale effects, and limitations of instruments used to capture data of model behavior.

In maneuvering tests with free-running models, the propeller is used to give the desired model speed, i.e. to produce the thrust to keep the desired speed. Scale effects may generally be neglected, at least for conventional displacement vessels with a propeller working in the wake of the hull and the rudder positioned in the propeller slipstream. Fortunately, two phenomena - the larger model wake fraction and the larger model resistance - tend to even out in the rudder force. As a result of these scale effects, rudder effectiveness of a model may generally be over-estimated compared with that of a real ship. Accordingly, free models tend to be more stable with respect to the course-keeping ability.

Through these simulations, it is obvious that the mathematical model is compatible with what one expects from a real ship. It shows that the system identification method can be applied to determine the hydrodynamic coefficients not only precisely but also in a manner that is adoptable to real ship maneuvers. Besides all, the advantage of the method as a lowcost procedure with a short time makes it much powerful in the simulation of ship maneuvering based on experimental data. The method facilitates the procedure of hydrodynamic coefficients determination when compared to other similar methods as it represents all the coefficients simultaneously through a fast procedure. Finally, it should be noted that when compared to complicated theoretical approaches or captive model tests, the system identification method based on free-running model test seems to be so promising.

\section{Conclusion}

Three degrees of freedom of mathematical model are represented based on ship hydrodynamic analysis. The system identification theory is applied for estimation of model hydrodynamic coefficients through an algorithm based on Extended Kalman Filter. In order to obtain experimental data as input of extended Kalman filter method, free-running model tests of a 4.36-m model of KCS are performed in open lake and different maneuvering tests are executed. Ship velocities in directions of surge, sway, and yaw are measured and this data is utilized as input of extended Kalman filter algorithms. Finally, hydrodynamic coefficients are determined. Then, different maneuvers are simulated through mathematical model and the motion trajectories are obtained by integration of corresponding velocities. The turning maneuvering is simulated and compared to experimental data from free-running model test for validation of the procedure. It seems that the developed model can estimate ship maneuvering performance with enough accuracy when compared to model test results. According to the results, the developed mathematical model can reflect the real ship maneuvering characteristics with acceptable accuracy. The results show that the procedure and algorithm developed for hydrodynamic coefficients estimation are applicable and in advance can be used in ship maneuvering simulation and trajectory control. It should be emphasized that the developed method in this paper is independent of the form of mathematical model and it is possible to determine different coefficients in various equations.

\section{References}

1. Wang, X., Zou, Z. and Yin, J. "Modular parameter identification for ship maneuvering prediction based on support vector machines", Proceedings of the TwentyThird International Offshore and Polar Engineering, pp. 834-839 (2013).

2. Ross, A., Perez, T. and Fossen, T. "A novel maneuvering model based on low-aspect-ratio lift theory and Lagrangian mechanics", In: Proceedings of the IFAC Conference on Control Applications in Marine System (CAMS), pp. 1-4 (2007).

3. Ogawa, A. and Kasai, H. "On the mathematical model of manoeuvring motion of ship", International Shipbuilding Progress, 25(292), pp. 306-319 (1978).

4. Stern, F., Agdrup, K., Kim, S.Y., Hochbaum, A.C., Rhee, K.P., Quadvlieg, F., Perdon, P., Hino, T., Broglia, R. and Gorski, J. "Experience from SIMMAN 2008-the first workshop on verification and validation of ship maneuvering simulation methods", Journal of Ship Research, 55(2), pp. 135-147 (2011).

5. Shi, C. "Collaboration to enhance development and application of shiphandling simulators", International symposium on GPS/GNSS, 12, pp. 459-464 (2006).

6. Dianpu, L. "Ship motion modeling", Harbin Engineering, pp. 1-90, University Press, Harbin (1999).

7. Lewis, F.L., Optimal Estimation with an Introduction to Stochastic Control Theory, Publishing by John Wiley \& Sons Company, pp. 1-10 (1986).

8. Kalman, R.E. "A new approach to linear filtering and prediction problems", Transactions of the ASME, Journal of Basic Engineering, 82 (Series D), pp. 35-45 (1960).

9. Farina A. "Tracking a ballistic target: Comparison of several nonlinear filters", IEEE Transactions on Aero-space and Electronic Systems, 38(3), pp. 85-867 (2002).

10. The Manoeuvring Committee, Final Report and Recommendations to the 24th ITTC, pp.1-8, UK (2005). 
11. Abkowitz, M.A. "Measurement of hydrodynamic characteristics from ship maneuvering trials by system identification", Proceedings of the Annual Meeting of SNAM, pp. 1-27 (1980).

12. Rhee, K.P. and Kim, K. "A new sea trial method for estimating hydrodynamic derivatives", Ship \& Ocean Technology, 3(3), pp. 25-44 (1999).

13. The Manoeuvring Committee "Final report and recommendations to the 23rd ITTC", pp. 1-7, 23rd International Towing Tank Conference (2002).

14. Xin-guang, Z. and Zao-jian, Z. "Identification of Abkowitz model for ship manoeuvring motion using $\varepsilon$ support vector regression", Journal of Hydrodynamics, 23(3), pp. 353-360 (2011).

15. Lokukaluge, P., Oliveira, P. and Soares, C. "System identification of nonlinear vessel steering", Journal of Offshore Mechanics and Arctic Engineering, 137, pp. 1-7 (2015).

16. Lewandowski, M. "The dynamics of marine craft: Maneuvering and Seakeeping", Advanced Series on Ocean Engineering, 22, pp. 300-383, Copyright by World Scientific Publishing Co. Pte. Ltd (2004).

17. Karrari, M., System Identification, Amirkabir University of Technology, pp. 1-50, Tehran Polytechnic Press (2010).

\section{Appendix A}

\section{Extended Kalman filtering.}

The EKF is in a continuous cycle of predict-update. The following lists the equations for the EKF:

1. Predict next state:

$$
X_{t, t-1}=f\left(X_{t-1, t-1}, 0\right),
$$

where $f\left(X_{t-1, t-1}, 0\right)$ is the approximated state $\tilde{x}_{t}$.

2. Predict next state covariance:

$$
S_{t, t-1}=\left(\frac{\partial f}{\partial x}\right) S_{t, t-1}\left(\frac{\partial f}{\partial x}\right)^{T}+\left(\frac{\partial f}{\partial a}\right) Q\left(\frac{\partial f}{\partial a}\right)_{(\mathrm{A} .2)}^{T}
$$

where $\frac{\partial f}{\partial x}$ and $\frac{\partial f}{\partial a}$ are the Jacobians of the state transition equations. The notation $(\ldots)^{T}$ indicates matrix transpose.

3. Obtain measurement(s) $Y_{t}$.

4. Calculate the Kalman gain (weights):

$$
\begin{aligned}
K_{t}= & S_{t, t-1}\left(\frac{\partial g}{\partial x}\right)^{T}\left[\left(\frac{\partial g}{\partial x}\right) S_{t, t-1}\left(\frac{\partial g}{\partial x}\right)^{T}\right. \\
& \left.+\left(\frac{\partial g}{\partial n}\right) R\left(\frac{\partial g}{\partial n}\right)^{T}\right]^{-1},
\end{aligned}
$$

where $\frac{\partial g}{\partial x}$ and $\frac{\partial g}{\partial n}$ are the Jacobians of the measurement equations.
5. Update state:

$$
X_{t, t}=X_{t, t-1}+K_{t}\left[Y_{t}-g\left(\tilde{x}_{t}, 0\right)\right] \text {, }
$$

where $g\left(\tilde{x}_{t}, 0\right)$ is the ideal (noiseless) measurement of the approximated state above.

6. Update state covariance:

$$
S_{t, t}=\left[I-K_{t}\left(\frac{\partial g}{\partial x}\right)\right] S_{t, t-1} .
$$

7. Loop (now $t$ becomes $t+1$ ):

In order to understand this concept, we will look at an example. Consider tracking an object in 2D using a constant velocity model. Thus, the state variables are $X$ and $Y$ position and velocity:

$$
X_{t}=\left[\begin{array}{c}
x_{t} \\
\dot{x}_{t} \\
y_{t} \\
\dot{y}_{t}
\end{array}\right]
$$

The state transition equations for this model are:

$$
f\left(x_{t}, a_{t}\right)=\left[\begin{array}{c}
x_{t+1}=x_{t}+T \dot{x}_{t}+0 \\
\dot{x}_{t+1}=\dot{x}_{t}+u_{1} \\
y_{t+1}=y_{t}+T \dot{y}_{t}+0 \\
\dot{y}_{t+1}=\dot{y}_{t}+u_{2}
\end{array}\right]
$$

where $u_{1}$ and $u_{2}$ are random samples drawn from $N\left(0, \sigma_{a}^{2}\right)$, representing an unknown acceleration.

For observations, consider using a sensor that operates on polar coordinates, providing an $r$ and $\theta$ measurement:

$$
Y_{t}=\left[\begin{array}{l}
r_{t} \\
\theta_{t}
\end{array}\right]
$$

The observation equations for this model are:

$$
g\left(x_{t}, n_{t}\right)=\left[\begin{array}{c}
r_{t}=\sqrt{x_{t}^{2}+y_{t}^{2}}+n_{1} \\
\theta_{t}=\tan ^{-1} \frac{y_{t}}{x_{t}}+n_{2}
\end{array}\right]
$$

where $n_{1}$ is a random sample drawn from $N\left(0, \sigma_{\text {dist }}^{2}\right)$ and $n_{2}$ is a random sample drawn from $N\left(0, \sigma_{d i r}^{2}\right)$, representing noises on the measured distance and direction, respectively.

In order to use this model in the EKF, we must calculate the four Jacobians. The derivative of the state transition equations with respect to the state variables is: 


$$
\frac{\partial f}{\partial x}=\frac{\partial f}{\partial(x, \dot{x}, y, \dot{y})}=\left[\begin{array}{cccc}
1 & T & 0 & 0 \\
0 & 1 & 0 & 0 \\
0 & 0 & 1 & T \\
0 & 0 & 0 & 1
\end{array}\right]
$$

The derivative of the state transition equations with respect to the dynamic noises is:

$$
\frac{\partial f}{\partial a}=\frac{\partial f}{\partial\left(0, u_{1}, 0, u_{2}\right)}=\left[\begin{array}{cccc}
0 & 0 & 0 & 0 \\
0 & 1 & 0 & 0 \\
0 & 0 & 0 & 0 \\
0 & 0 & 0 & 1
\end{array}\right]
$$

The Jacobians of both the state transition equations are fairly simple, because that portion of this model is linear. Therefore, all the derivatives are constant.

The derivative of the observation equations with respect to the state variables is:

$$
\begin{aligned}
\frac{\partial f}{\partial x} & =\frac{\partial f}{\partial(x, \dot{x}, y, \dot{y})} \\
& =\left[\begin{array}{cccc}
\frac{\partial}{\partial} \sqrt{x^{2}+y^{2}}+n_{1} & 0 & \frac{\partial}{\partial} \sqrt{x^{2}+y^{2}}+n_{1} & 0 \\
\frac{\partial}{\partial} \tan ^{-1} \frac{y}{x}+n_{2} & 0 & \frac{\partial}{\partial} \tan ^{-1} \frac{y}{x}+n_{2} & 0
\end{array}\right] \\
& \left.=\left[\begin{array}{cccc}
y\left(x^{2}+y^{2}\right)^{-1 / 2} & 0 & y\left(x^{2}+y^{2}\right)^{-1 / 2} & 0 \\
\frac{y}{x^{2}+y^{2}} & 0 & \frac{y}{x^{2}+y^{2}} & 0
\end{array}\right] . \text { (A.1 }\right)
\end{aligned}
$$

The equation makes use of the functions for the derivative of arctan, the power rule, and the chain rule. The time subscripts are omitted for clarity. However, in practice, the values in the matrix $\frac{\partial g}{\partial x}$ must be calculated in every iteration. They are calculated using values from the current filtered estimate of the state variables. The derivative of the observation equations with respect to the measurement noises is:

$$
\frac{\partial g}{\partial n}=\frac{\partial g}{\partial\left(n_{1}, n_{2}\right)}=\left[\begin{array}{ll}
1 & 0 \\
0 & 1
\end{array}\right] \text {. }
$$

To finish this example, we must look at the covariances. The covariance of the dynamic noises is:

$$
Q=\left[\begin{array}{cccc}
0 & 0 & 0 & 0 \\
0 & \sigma_{a}^{2} & 0 & \text { small } \neq \\
0 & 0 & 0 & 0 \\
0 & \text { small } \neq & 0 & \sigma_{a}^{2}
\end{array}\right],
$$

where a small number allows for some covariance in practice. The covariance of the measurement noises is:

$$
R=\left[\begin{array}{cc}
\sigma_{d i s}^{2} & \text { small } \neq \\
\text { small } \neq & \sigma_{d i r}^{2}
\end{array}\right] .
$$

The covariance of the state, $S$, is a $4 \times 4$ matrix with the variances of $x, \dot{x}, y, \dot{y}$ along the diagonal and the (hopefully very small) covariances in the other elements.

Finally, in order to verify that the problem has been configured properly, one should go through all the EKF equations above and make sure that the matrix sizes match.

Doing this will reveal that the Kalman gain matrix $K$ is $4 \times 2$, and everything fits:

$$
\begin{aligned}
& \overbrace{X}^{4 \times 1}=\overbrace{f}^{4 \times 1} \\
& \overbrace{S}^{4 \times 4}=\overbrace{\left(\frac{\partial f}{\partial x}\right)}^{4 \times 4} \overbrace{S}^{4 \times 4} \overbrace{\left(\frac{\partial f}{\partial x}\right)^{T}}^{4 \times 4}+\overbrace{\left(\frac{\partial f}{\partial a}\right)^{2}}^{4 \times 4} \overbrace{Q}^{4 \times 4} \overbrace{\left(\frac{\partial f}{\partial a}\right)^{T}}^{4 \times 4}, \\
& \overbrace{K}^{4 \times 2}=\overbrace{S}^{4 \times 4} \overbrace{\left(\frac{\partial g}{\partial x}\right)^{T}}^{4 \times 2}[\overbrace{\left(\frac{\partial g}{\partial x}\right)^{2}}^{2 \times 4} \overbrace{S}^{4 \times 4} \overbrace{\left(\frac{\partial g}{\partial x}\right)^{T}}^{4 \times 2} \\
& +\overbrace{\left(\frac{\partial g}{\partial n}\right)}^{2 \times 2} \overbrace{R}^{2 \times} \overbrace{\left(\frac{\partial g}{\partial n}\right)^{T}}^{2 \times 2}]^{-1} \\
& \overbrace{X}^{4 \times 1}=\overbrace{X}^{4 \times 1}+\overbrace{K}^{4 \times 2}(\overbrace{Y}^{2 \times 1}-\overbrace{g}^{2 \times 1}), \\
& \overbrace{S}^{4 \times 4}=[\overbrace{I}^{4 \times 4}-\overbrace{K}^{4 \times 2} \overbrace{\left(\frac{\partial g}{\partial x}\right)}^{2 \times 4}] \overbrace{S_{t, t-1}}^{4 \times 4} .
\end{aligned}
$$

\section{Biographies}

Sajad Hajizadeh received the BSc degree in Naval Architecture from Persian Gulf University, Busher, Iran, in 2007, and the MSc degree in Marine Engineering from Sharif University of Technology, Tehran, Iran, in 2010. He is currently is currently $\mathrm{PhD}$ student in School of Mechanical Engineering at Sharif University of Technology. His main area of interest is hydrodynamics and maneuvering of marine vehicles. He has published several papers in this field.

Mohammad Saeed Seif received the BSc degree in Mechanical Engineering from Amirkabir University of Technology, Tehran, Iran, in 1989; the MSc degree in Naval Architecture from Technical University of Gdansk, Gdansk, Poland, in 1992; and PhD degree in Mechanical Engineering from Yokohama National University, Yokohama, Japan, in 1995. He is currently 
Professor in the Department of Mechanical Engineering, Sharif University of Technology, Tehran, Iran. His area of expertise is dynamics and hydrodynamics of marine vehicles, ship structure design, advanced marine vehicles, and advanced fluid dynamics. He has published several papers in the maneuvering field.

Hamid Mehdigholi received the BS degree in Me- chanical Engineering from Sharif University, Tehran, Iran, in 1977, and the MS and PhD degrees in Applied Mechanics from Imperial College, London, in 1986 and 1991, respectively. He has been a faculty member at Sharif University of Technology since 1991. His research interests include dynamics of rotating machines, vibration isolation, acoustics, and experimental modal analysis. 